\title{
An Energy Inequality and Its Applications of Nonlocal Boundary Conditions of Mixed Problem for Singular Parabolic Equations in Nonclassical Function Spaces
}

\author{
Moussa Zakari Djibibe, ${ }^{1}$ Kokou Tcharie, ${ }^{1}$ \\ and N. Iossifovich Yurchuk ${ }^{2}$ \\ ${ }^{1}$ Department of Mathematics, University of Lomé, P.O. Box 1515, Lomé, Togo \\ ${ }^{2}$ Faculty of Mechanics and Mathematics, Belarusian State University, Minsk 220050, Belarus \\ Correspondence should be addressed to Moussa Zakari Djibibe, zakari.djibibe@gmail.com
}

Received 23 July 2012; Accepted 14 August 2012

Academic Editors: C. Lu and E. Skubalska-Rafajlowicz

Copyright (C) 2012 Moussa Zakari Djibibe et al. This is an open access article distributed under the Creative Commons Attribution License, which permits unrestricted use, distribution, and reproduction in any medium, provided the original work is properly cited.

The aim of this paper is to establish a priori estimates of the following nonlocal boundary conditions mixed problem for parabolic equation: $\partial v / \partial t-\left(a(t) / x^{2}\right)(\partial / \partial x)\left(x^{2} \partial v / \partial x\right)+b(x, t) v=$ $g(x, t), v(x, 0)=\psi(x), 0 \leq x \leq \ell, v(\ell, t)=E(t), 0 \leq t \leq T, \int_{0}^{\ell} x^{3} v(x, t) d x=G(t), 0 \leq t \leq \ell$. It is important to know that a priori estimates established in nonclassical function spaces is a necessary tool to prove the uniqueness of a strong solution of the studied problems.

\section{Introduction}

In this paper, we deal with a class of parabolic equations with time- and space-variable characteristics, with a nonlocal boundary condition. The precise statement of the problem is a follows: let $\ell>0, T>0$, and $\Omega=\left\{(x, t) \in \mathbb{R}^{2}: 0<x<\ell, 0<t<T\right\}$. We will determine a solution $v$, in $\Omega$ of the differential equation

$$
\frac{\partial v}{\partial t}-\frac{a(t)}{x^{2}} \frac{\partial}{\partial x}\left(x^{2} \frac{\partial v}{\partial x}\right)+b(x, t) v=g(x, t), \quad(x, t) \in \Omega,
$$

satisfying the initial condition

$$
v(x, 0)=\psi(x), \quad 0 \leq x \leq \ell,
$$


the classical condition

$$
v(\ell, t)=E(t), \quad 0 \leq t \leq T
$$

and the integral condition

$$
\int_{0}^{\ell} x^{3} v(x, t) d x=G(t), \quad 0 \leq t \leq T .
$$

For consistency, we have

$$
\int_{0}^{\ell} x^{3} \psi(x)=G(0), \quad \psi(\ell)=E(0),
$$

where $\ell$ and $T$ are fixed but arbitrary positive numbers, $a(t)$ and $b(x, t)$ are the known fuctions satisfying the following condition.

Condition 1. For $t \in[0, T]$ and $x \in[0, \ell]$, we assume that

(i) $d_{0} \leq a(t) \leq d_{1}$,

(ii) $b(x, t) \leq d_{2}$,

(iii) $d a(t) / d t \leq d_{3}$.

The notion of nonlocal condition has been introduced to extend the study of the classical initial value problems and it is more precise for describing natural phenomena than the classical condition since more information is taken into account, thereby decreasing the negative effects incurred by a possibly erroneous single measurement taken at the initial value. The importance of nonlocal conditions in many applications is discussed in [1,2].

It can be a part in the contribution of the development of a priori estimates method for solving such problems. The questions related to these problems are so miscellaneous that the elaboration of a general theory is still premature. Therefore, the investigation of these problems requires at every time a separate study.

This work can be considered as a continuation of the results of Yurchuk [3], Benouar and Yurchuk [4], Bouziani [5-7], Bouziani and Benouar [8], Djibibe et al. [9], and Djibibe and Tcharie [10]. Our results generalize and deepen ones from corresponding work in [11,12].

We should mention here that the presence of an integral term in the boundary condition can greatly complicate the application of standard functional and numerical techniques.

This paper is organized as follows. After this introduction, in Section 2, we present the preliminaries. Finally, in Section 3, we establish an energy inaquality and give its several applications. 


\section{Preliminares}

We transform the problem with nonhomogeneous boundary conditions into a problem with homogeneous boundary conditions. For this, we introduce a new unknown function $u$ defined by $v(x, t)=u(x, t)+w(x, t)$, where

$$
w(x, t)=\left(\frac{5 x}{\ell}-4\right) E(t)-\frac{20}{\ell^{5}}(x-\ell) G(t) .
$$

Then, problem becomes

$$
\begin{gathered}
\frac{\partial u}{\partial t}-\frac{a(t)}{x^{2}} \frac{\partial}{\partial x}\left(x^{2} \frac{\partial u}{\partial x}\right)+b(x, t) u=f(x, t), \\
u(x, 0)=\varphi(x), \quad 0 \leq x \leq \ell, \\
u(\ell, t)=0, \quad 0 \leq t \leq T, \\
\int_{0}^{\ell} x^{3} u(x, t) d x=0, \quad 0 \leq t \leq \ell
\end{gathered}
$$

where

$$
\begin{aligned}
\varphi(x)= & \psi(x)+\frac{20}{\ell}(x-\ell) G(0)-\frac{1}{\ell}(5 x-4 \ell) E(0) \\
f(x, t)= & F(x, t)+\frac{20}{\ell^{5}}(x-\ell)\left(b(x, t) G(t)+G^{\prime}(t)\right)-\frac{1}{\ell}(5 x-4 \ell)\left(b(x, t) E(t)+E^{\prime}(t)\right) \\
& +\frac{10}{\ell^{5}} x a(t)\left(\ell^{4} E(t)-G(t)\right) .
\end{aligned}
$$

We introduce appropriate function spaces. Let $L^{2}(\Omega)$ be the Hilbert space of square integrable functions. To problem (2.1), (2.2), (2.3), (2.5), we associate the operator $A$ with the domain of definition

$$
D(A)=\left\{\frac{\partial u}{\partial t}, \frac{1}{x^{2}} \frac{\partial u}{\partial x}, \frac{\partial^{2} u}{\partial x^{2}} \in L^{2}(\Omega)\right\}
$$

satisfying (2.4) and (2.5). The operator $A$ is considered from $E$ to $F$, where $E$ is the banach space consisting of $u \in L^{2}(\Omega)$ satisfying the boundary conditions (2.4) and (2.5) and having the finite norm:

$$
\|u\|^{2}=\int_{\Omega} J_{x}^{2}\left(\frac{\partial u}{\partial t}\right) d x d t+\sup _{0 \leq t \leq T}\left\{\int_{0}^{\ell} x^{2} u^{2}(x, t) d x+\int_{0}^{\ell}\left(x \frac{\partial u}{\partial t}\right)^{2} d x+\int_{0}^{\ell}\left(x \frac{\partial u}{\partial x}\right)^{2} d x\right\},
$$


and $F$ is the Hilbert space of vector-value function $F=(f, \varphi)$ having the norm

$$
\|(f, \varphi)\|^{2}=\int_{\Omega} x^{2} f^{2}(x, t) d x d t+\int_{0}^{\ell} x^{2} \varphi^{2}(x) d x+\int_{0}^{\ell}\left(x \frac{\partial \varphi(x)}{\partial x}\right)^{2} d x
$$

where $J_{x} h=\int_{x}^{\ell} \theta^{2} h(\theta, t) d \theta$.

\section{A Priori Estimate and Its Consequences}

Theorem 3.1. Under Condition 1, for any function $v \in D(A)$, one has the following a priori estimate

$$
\|v\|_{E} \leq c\|A v\|_{F}
$$

where $c$ is a positive constant independent of the solution $v$.

Proof. Firstly, applying operator $J_{x}$ to (2.1), multiplying the obtained result with $J_{x}(\partial u / \partial t)$, and integrating over $\Omega_{\tau}=(0, \ell) \times(0, \tau)$, oberve that

$$
\begin{aligned}
& \int_{\Omega_{\tau}} J_{x}^{2}\left(\frac{\partial u}{\partial t}\right) d x d t-\int_{\Omega_{\tau}} J_{x}\left(\frac{a(t)}{x^{2}} \frac{\partial}{\partial x}\left(x^{2} \frac{\partial u}{\partial x}\right)\right) J_{x}\left(\frac{\partial u}{\partial t}\right) d x d t \\
& \quad+\int_{\Omega_{\tau}} J_{x}(b(x, t) u) J_{x}\left(\frac{\partial u}{\partial t}\right) d x d t=\int_{\Omega_{\tau}} J_{x}(f(x, t)) J_{x}\left(\frac{\partial u}{\partial t}\right) d x d t .
\end{aligned}
$$

Integrating by parts of the second integral on the left-hand side of (3.2), we get

$$
-\int_{\Omega_{\tau}} J_{x}\left(\frac{a(t)}{x^{2}} \frac{\partial}{\partial x}\left(x^{2} \frac{\partial u}{\partial x}\right)\right) J_{x}\left(\frac{\partial u}{\partial t}\right) d x d t=\int_{\Omega_{\tau}} x^{2} a(t) \frac{\partial u}{\partial x} J_{x}\left(\frac{\partial u}{\partial t}\right) d x d t
$$

Substituting (3.3) into (3.2), we get

$$
\begin{aligned}
& \int_{\Omega_{\tau}} J_{x}^{2}\left(\frac{\partial u}{\partial t}\right) d x d t+\int_{\Omega_{\tau}} x^{2} a(t) \frac{\partial u}{\partial x} J_{x}\left(\frac{\partial u}{\partial t}\right) d x d t \\
& \quad+\int_{\Omega_{\tau}} J_{x}(b(x, t) u) J_{x}\left(\frac{\partial u}{\partial t}\right) d x d t=\int_{\Omega_{\tau}} J_{x}(f(x, t)) J_{x}\left(\frac{\partial u}{\partial t}\right) d x d t
\end{aligned}
$$

In the second time, multiplying the equality (2.1) with $x^{2} \partial u / \partial t$, and integrating the obtained equality over $\Omega_{\tau}$, we get

$$
\begin{aligned}
& \int_{\Omega_{\tau}}\left(x \frac{\partial u}{\partial t}\right)^{2} d x d t-\int_{\Omega_{\tau}} a(t) \frac{\partial}{\partial x}\left(x^{2} \frac{\partial u}{\partial x}\right) \frac{\partial u}{\partial t} d x d t \\
& \quad+\int_{\Omega_{\tau}} x^{2} b(x, t) u \frac{\partial u}{\partial t} d x d t=\int_{\Omega_{\tau}} x^{2} f(x, t) \frac{\partial u}{\partial t} d x d t
\end{aligned}
$$


The standard integration by parts of the second term on the left-hand side of (3.5), leads to

$$
\begin{aligned}
-\int_{\Omega_{\tau}} a(t) \frac{\partial}{\partial x}\left(x^{2} \frac{\partial u}{\partial x}\right) \frac{\partial u}{\partial t} d x d t= & \frac{1}{2} \int_{0}^{\ell} a(\tau) x^{2}\left(\frac{\partial u}{\partial x}(x, \tau)\right)^{2} d x-\frac{1}{2} \int_{0}^{\ell} a(0) x^{2}\left(\frac{\partial \varphi}{\partial x}\right)^{2} \\
& -\frac{1}{2} \int_{\Omega_{\tau}} a^{\prime}(t) x^{2}\left(\frac{\partial u}{\partial x}\right)^{2} d x d t .
\end{aligned}
$$

Substituting (3.6) into (3.5), we get

$$
\begin{aligned}
\int_{\Omega_{\tau}} & \left(x \frac{\partial u}{\partial t}\right)^{2} d x d t+\frac{1}{2} \int_{0}^{\ell} a(\tau) x^{2}\left(\frac{\partial u}{\partial x}(x, \tau)\right)^{2} d x-\frac{1}{2} \int_{0}^{\ell} a(0) x^{2}\left(\frac{\partial \varphi}{\partial x}\right)^{2} \\
& -\frac{1}{2} \int_{\Omega_{\tau}} a^{\prime}(t) x^{2}\left(\frac{\partial u}{\partial x}\right)^{2} d x d t+\int_{\Omega_{\tau}} x^{2} b(x, t) u \frac{\partial u}{\partial t} d x d t=\int_{\Omega_{\tau}} x^{2} f(x, t) \frac{\partial u}{\partial t} d x d t .
\end{aligned}
$$

Finally, adding (3.4) to (3.7), we have

$$
\begin{aligned}
\int_{\Omega_{\tau}} J_{x}^{2}\left(\frac{\partial u}{\partial t}\right) d x d t+\int_{\Omega_{\tau}}\left(x \frac{\partial u}{\partial t}\right)^{2} d x d t+\frac{1}{2} \int_{0}^{\ell} a(\tau) x^{2}\left(\frac{\partial u}{\partial x}(x, \tau)\right)^{2} d x \\
=\int_{\Omega_{\tau}} J_{x}(f(x, t)) J_{x}\left(\frac{\partial u}{\partial t}\right) d x d t+\int_{\Omega_{\tau}} x^{2} f(x, t) \frac{\partial u}{\partial t} d x d t+\frac{1}{2} \int_{0}^{\ell} a(0) x^{2}\left(\frac{\partial \varphi}{\partial x}\right)^{2} \\
\quad-\int_{\Omega_{\tau}} x^{2} b(x, t) u \frac{\partial u}{\partial t} d x d t-\int_{\Omega_{\tau}} J_{x}(b(x, t) u) J_{x}\left(\frac{\partial u}{\partial t}\right) d x d t \\
+\frac{1}{2} \int_{\Omega_{\tau}} a^{\prime}(t) x^{2}\left(\frac{\partial u}{\partial x}\right)^{2} d x d t-\int_{\Omega_{\tau}} x^{2} a(t) \frac{\partial u}{\partial x} J_{x}\left(\frac{\partial u}{\partial t}\right) d x d t .
\end{aligned}
$$

In the light of Cauchy inequality, certain terms of (3.8) are then majorized as follows:

$$
\begin{gathered}
\int_{\Omega_{\tau}} J_{x}(f(x, t)) J_{x}\left(\frac{\partial u}{\partial t}\right) d x d t \leq \frac{\alpha_{1}}{2} \int_{\Omega_{\tau}} J_{x}^{2}(f(x, t)) d x d t+\frac{1}{2 \alpha_{1}} \int_{\Omega_{\tau}} J_{x}^{2}\left(\frac{\partial u}{\partial t}\right) d x d t \\
\int_{\Omega_{\tau}} x^{2} f(x, t) \frac{\partial u}{\partial t} d x d t \leq \frac{\alpha_{2}}{2} \int_{\Omega_{\tau}} x^{2} f^{2}(x, t) d x d t+\frac{1}{2 \alpha_{2}} \int_{\Omega_{\tau}}\left(x \frac{\partial u}{\partial t}\right)^{2} d x d t \\
-\int_{\Omega_{\tau}} x^{2} a(t) \frac{\partial u}{\partial x} J_{x}\left(\frac{\partial u}{\partial t}\right) d x d t \leq \frac{\alpha_{3}}{2} \int_{\Omega_{\tau}} a^{2}(t)\left(x \frac{\partial u}{\partial x}\right)^{2} d x d t+\frac{1}{2 \alpha_{3}} \int_{\Omega_{\tau}} J_{x}^{2}\left(\frac{\partial u}{\partial t}\right) d x d t \\
-\int_{\Omega_{\tau}} x^{2} b(x, t) u \frac{\partial u}{\partial t} d x d t \leq \frac{\alpha_{4}}{2} \int_{\Omega_{\tau}} x^{2} b^{2}(x, t) u^{2}(x, t) d x d t+\frac{1}{2 \alpha_{4}} \int_{\Omega_{\tau}}\left(x \frac{\partial u}{\partial t}\right)^{2} d x d t \\
-\int_{\Omega_{\tau}} J_{x}(b(x, t) u) J_{x}\left(\frac{\partial u}{\partial t}\right) d x d t \leq \frac{\alpha_{5}}{2} \int_{\Omega_{\tau}} J_{x}^{2}(b(x, t) u) d x d t+\frac{1}{2 \alpha_{5}} \int_{\Omega_{\tau}} J_{x}^{2}\left(\frac{\partial u}{\partial t}\right) d x d t
\end{gathered}
$$


Combining the inequalities (3.9), (3.10), (3.11) with (3.8), choosing $\alpha_{1}, \alpha_{2}, \alpha_{3}, \alpha_{4}, \alpha_{5}$ which that $\alpha_{1}+\alpha_{3}+\alpha_{5}<2 \alpha_{1} \alpha_{3} \alpha_{5}$ and $\alpha_{2}+\alpha_{4}<2 \alpha_{2} \alpha_{4}$, we get

$$
\begin{aligned}
\lambda_{1} \int_{\Omega_{\tau}} & J_{x}^{2}\left(\frac{\partial u}{\partial t}\right) d x d t+\lambda_{2} \int_{\Omega_{\tau}}\left(x \frac{\partial u}{\partial t}\right)^{2} d x d t+\frac{1}{2} \int_{0}^{\ell} a(\tau)\left(x \frac{\partial u}{\partial x}(x, \tau)\right)^{2} d x \\
\leq & \frac{1}{2} \int_{\Omega_{\tau}}\left(\alpha_{3} a^{2}(t)+a^{\prime}(t)\right)\left(x \frac{\partial u}{\partial x}\right)^{2} d x d t+\frac{\alpha_{4}}{2} \int_{\Omega_{\tau}} x^{2} b^{2}(x, t) u^{2}(x, t) d x d t \\
& +\frac{\alpha_{5}}{2} \int_{\Omega_{\tau}} J_{x}^{2}(b(x, t) u) d x d t+\frac{\alpha_{1}}{2} \int_{\Omega_{\tau}} J_{x}^{2}(f(x, t)) d x d t \\
& +\frac{\alpha_{2}}{2} \int_{\Omega_{\tau}} x^{2} f^{2}(x, t) d x d t+\frac{1}{2} \int_{0}^{\ell} a(0)\left(x \frac{\partial \varphi}{\partial x}\right)^{2},
\end{aligned}
$$

where

$$
\lambda_{1}=1-\frac{1}{2}\left(\frac{1}{\alpha_{1}}+\frac{1}{\alpha_{3}}+\frac{1}{\alpha_{5}}\right) \quad \lambda_{2}=1-\frac{1}{2}\left(\frac{1}{\alpha_{2}}+\frac{1}{\alpha_{4}}\right)
$$

Lemma 3.2. For $x \in(0, \ell)$, the following inequalities hold:

$$
\begin{gathered}
\int_{\Omega_{\tau}} J_{x}^{2}(u) d x d t \leq \frac{\ell^{2}}{2} \int_{0}^{\ell} x^{2} u^{2} d x \\
\lambda_{2} \int_{0}^{\ell} x^{2} u^{2} d x \leq \lambda_{2} \int_{0}^{\ell} x^{2} \varphi^{2}(x) d x+\lambda_{2} \int_{\Omega_{\tau}} x^{2} u^{2}(x, t) d x d t+\lambda_{2} \int_{\Omega_{\tau}}\left(x \frac{\partial u}{\partial t}\right)^{2} d x d t .
\end{gathered}
$$

It follows by using Lemma 3.2 and (3.18) that

$$
\begin{aligned}
\lambda_{1} \int_{\Omega_{\tau}} J_{x}^{2}\left(\frac{\partial u}{\partial t}\right) d x d t+\frac{1}{2} \int_{0}^{\ell} a(\tau)\left(x \frac{\partial u}{\partial x}(x, \tau)\right)^{2} d x+\frac{\lambda_{2}}{2} \int_{0}^{\ell} x^{2} u^{2} d x \\
\quad \leq \frac{1}{2} \int_{\Omega_{\tau}}\left(\alpha_{3} a^{2}(t)+a^{\prime}(t)\right)\left(x \frac{\partial u}{\partial x}\right)^{2} d x d t+\frac{\left(2 \alpha_{4}+\alpha_{5} \ell^{2}\right)}{4} \int_{\Omega_{\tau}} x^{2} b^{2}(x, t) u^{2}(x, t) d x d t \\
\quad+\frac{\alpha_{1} \ell^{2}+2 \alpha_{2}}{4} \int_{\Omega_{\tau}} x^{2} f^{2}(x, t) d x d t+\frac{\lambda_{2}}{2} \int_{0}^{\ell} x^{2} \varphi^{2}(x) d x+\frac{1}{2} \int_{0}^{\ell} a(0)\left(x \frac{\partial \varphi}{\partial x}\right)^{2} .
\end{aligned}
$$


Therefore, by formula (3.17) and Condition 1, we obtain

$$
\begin{aligned}
\int_{\Omega_{\tau}} J_{x}^{2} & \left(\frac{\partial u}{\partial t}\right) d x d t+\int_{0}^{\ell}\left(x \frac{\partial u}{\partial x}(x, \tau)\right)^{2} d x+\int_{0}^{\ell} x^{2} u^{2} d x \\
\leq & \lambda_{3}\left(\int_{\Omega_{\tau}} x^{2} f^{2}(x, t) d x d t+\int_{0}^{\ell} x^{2} \varphi^{2}(x) d x+\int_{0}^{\ell}\left(x \frac{\partial \varphi}{\partial x}\right)^{2} d x\right) \\
& +\lambda_{4}\left(\int_{\Omega_{\tau}}\left(x \frac{\partial u}{\partial x}\right)^{2} d x d t+\int_{\Omega_{\tau}} x^{2} u^{2}(x, t) d x d t\right),
\end{aligned}
$$

where

$$
\lambda_{3}=\frac{\max \left(\left(\alpha_{1} \ell^{2}+2 \alpha_{2}\right) / 4, \lambda_{2} / 2, d_{1} / 2\right)}{\min \left(\lambda_{1}, \lambda_{2} / 2, d_{0} / 2\right)}, \quad \lambda_{4}=\frac{\max \left(\left(\alpha_{5} \ell^{2}+2 \alpha_{4}\right) d_{2}^{2} / 4, d_{3}+\alpha_{3} d_{1}^{2} / 2\right)}{\min \left(\lambda_{1}, \lambda_{2} / 2, d_{0} / 2\right)} .
$$

Eliminating the last term on the right-hand side of inequality (3.18). To this end, using Gronwall's lemma, it follows that

$$
\begin{aligned}
& \int_{\Omega_{\tau}} J_{x}^{2}\left(\frac{\partial u}{\partial t}\right) d x d t+\int_{0}^{\ell}\left(x \frac{\partial u}{\partial x}(x, \tau)\right)^{2} d x+\int_{0}^{\ell} x^{2} u^{2} d x \\
& \quad \leq \lambda_{5}\left(\int_{\Omega} x^{2} f^{2}(x, t) d x d t+\int_{0}^{\ell} x^{2} \varphi^{2}(x) d x+\int_{0}^{\ell}\left(x \frac{\partial \varphi}{\partial x}\right)^{2} d x\right),
\end{aligned}
$$

where $\lambda_{5}=\lambda_{3} e^{\lambda_{4} T}$.

The right-hand side of (3.20) is independent of $\tau$, hence, replacing the left-hand side by the upper bound with respect to $\tau$, We get

$$
\begin{aligned}
& \int_{\Omega} J_{x}^{2}\left(\frac{\partial u}{\partial t}\right) d x d t+\sup _{0 \leq t \leq T}\left\{\int_{0}^{\ell} x^{2} u^{2}(x, t) d x+\int_{0}^{\ell}\left(x \frac{\partial u}{\partial x}\right)^{2} d x\right\} \\
& \quad \leq c\left(\int_{\Omega} x^{2} f^{2}(x, t) d x d t+\int_{0}^{\ell} x^{2} \varphi^{2}(x) d x+\int_{0}^{\ell}\left(x \frac{\partial \varphi(x)}{\partial x}\right)^{2} d x\right),
\end{aligned}
$$

where $c=\sqrt{\lambda_{5}}=\sqrt{\lambda_{3} e^{\lambda_{4} T / 2}}$. This completes the proof of Theorem 3.1.

Lemma 3.3. The operator $A: E \rightarrow F$ with domain $D(A)$ has a closure $\bar{A}$.

Proof of Lemma 3.2. Suppose that $u_{n} \in D(A)$ is a sequence such that

$$
\begin{gathered}
\lim _{n \rightarrow+\infty} u_{n}=0, \quad \text { in } E, \\
\lim _{n \rightarrow+\infty} A u_{n}=(f, \varphi), \quad \text { in } F,
\end{gathered}
$$


we must show that $f \equiv 0$ and $\varphi \equiv 0$. Equality (3.22) implies that

$$
\lim _{n \rightarrow+\infty} u_{n}=0, \quad \text { in } \Phi^{\prime}(\Omega)
$$

By virtue of the condition of derivation of $\Phi^{\prime}(\Omega)$ in $\Phi^{\prime}(\Omega)$, we get

$$
\lim _{n \rightarrow+\infty}\left[\frac{\partial u_{n}}{\partial t}-a(x, t) \frac{\partial^{2} u_{n}}{\partial x^{2}}+b(x, t) \frac{\partial u_{n}}{\partial x}+c(x, t) u_{n}\right]=0, \quad \text { in } \Phi^{\prime}(\Omega)
$$

Then from equality (3.23) it follows that

$$
\lim _{n \rightarrow+\infty}\left[\frac{\partial u_{n}}{\partial t}-\frac{a(t)}{x^{2}} \frac{\partial}{\partial x}\left(x^{2} \frac{\partial u_{n}}{\partial x}\right)+b(x, t) u_{n}\right]=f, \quad \text { in } L^{2}(\Omega)
$$

therefore

$$
\lim _{n \rightarrow+\infty}\left[\frac{\partial u_{n}}{\partial t}-\frac{a(t)}{x^{2}} \frac{\partial}{\partial x}\left(x^{2} \frac{\partial u_{n}}{\partial x}\right)+b(x, t) u_{n}\right]=f, \quad \text { in } \Phi^{\prime}(\Omega)
$$

By virtue of the uniqueness of the limit in $\Phi^{\prime}(\Omega)$, the identies (3.25) and (3.27) conduct to $f \equiv 0$.

By analogy, from (3.23), we get

$$
\lim _{n \rightarrow+\infty} u_{n}(x, 0)=\varphi(x), \quad \text { in } L^{2}(0, \ell)
$$

We see via (3.22) and the obvious inequality

$$
\left\|u_{n}(x, 0)\right\|_{L^{2}(0, \ell)} \leq\left\|u_{n}(x, t)\right\|_{E^{\prime}}, \quad \forall n \in \mathbb{N}
$$

that

$$
\lim _{n \rightarrow+\infty} u_{n}(x, 0)=0, \quad \text { in } L^{2}(0, \ell)
$$

By virtue of (3.28), (3.30) and the uniqueness of the limit in $L^{2}(0, \ell)$ we conclude that $\varphi \equiv$ 0 .

Definition 3.4. A solution of the equation

$$
\bar{A} v=(f, \varphi),
$$

is called a strong solution of problem (2.2), (2.3), (2.4), and (2.5). 
Consequence 3.5. Under the conditions of Theorem 3.1, there is a constant $c>0$ independent of $v$ such that

$$
\|v\|_{E} \leq c\|\bar{A} v\|_{F^{\prime}} \quad \forall v \in D(\bar{A})
$$

Consequence 3.6. The range $R(\bar{A})$ of the operator $\bar{A}$ is closed and $\overline{R(A)}=\overline{R(A)}$.

Consequence 3.7. A strong solution of the problem (2.2), (2.3), (2.4), and (2.5) is unique and depends continuously on $\mathcal{F}=(f, \varphi) \in F$.

\section{References}

[1] A. A. Samarski1, "Some problems of the theory of differential equations," Differentsial'nye Uravneniya, vol. 16, no. 11, pp. 1925-1935, 1980.

[2] N. I. Yurchuk, "Solvability of boundary value problems for certain operator-differential equations," Differentsial'nye Uravneniya, vol. 13, no. 4, pp. 626-636, 1977.

[3] N. I. Yurchuk, "A mixed problem with an integral condition for some parabolic equations," Differentsial'nye Uravneniya, vol. 22, no. 12, pp. 2117-2126, 1986.

[4] N. E. Benouar and N. I. Yurchuk, "Mixed problem with an integral condition for parabolic equations with a Bessel operator," Differentsial'nye Uravneniya, vol. 27, no. 12, pp. 2094-2098, 1991.

[5] A. Bouziani, "Mixed problem for certain non-classical equations containing a small parameter," Académie Royale de Belgique, vol. 5, no. 7-12, pp. 389-400, 1994.

[6] A. Bouziani and N.-E. Benouar, "Problème mixte avec conditions intégrales pour une classe d'équations paraboliques," Comptes Rendus de l'Académie des Sciences, vol. 321, no. 9, pp. 1177-1182, 1995.

[7] A. Bouziani, "On the solvability of nonlocal pluriparabolic problem," Electronic Journal of Differential Equations, vol. 2001, no. 21, pp. 1-16, 2001.

[8] A. Bouziani and N.-E. Benouar, "Problème mixte avec conditions intégrales pour une classe d'équations paraboliques," Comptes Rendus de l'Académie des Sciences, vol. 321, no. 9, pp. 1177-1182, 1995 (French).

[9] M. Z. Djibibe, K. Tcharie, and N. I. Yurchuk, "Continuous dependence of solutions to mixed boundary value problems for a parabolic equation," Electronic Journal of Differential Equations, vol. 2008, pp. 1-10, 2008.

[10] M. Z. Djibibe and K. Tcharie, "Problème mixte pour une quation parabolique linèaire du deuxième ordre avec des conditions aux limites non locales en deux points," La Revue Ivoirienne des Sciences et Technologie, vol. 9, pp. 153-164, 2007.

[11] L. Bougoffa, "Parabolic equations with nonlocal conditions," Applied Mathematical Sciences, vol. 1, no. 21-24, pp. 1041-1048, 2007.

[12] A. Bouziani, "On the solvability of a class of singular parabolic equations with nonlocal boundary conditions in nonclassical function spaces," International Journal of Mathematics and Mathematical Sciences, vol. 30, no. 7, pp. 435-447, 2002. 


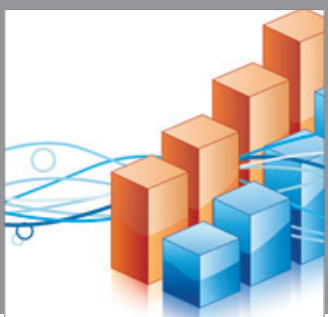

Advances in

Operations Research

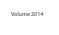

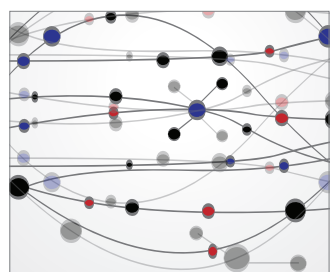

\section{The Scientific} World Journal
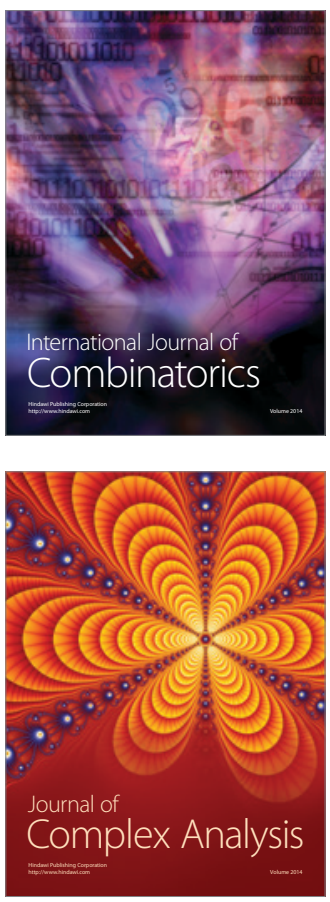

International Journal of

Mathematics and

Mathematical

Sciences
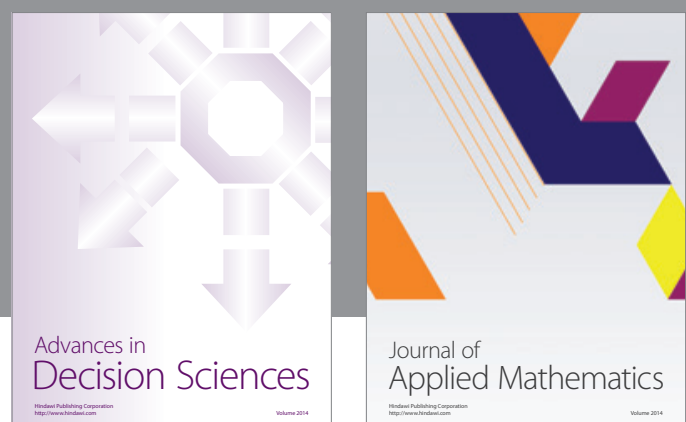

Journal of

Applied Mathematics
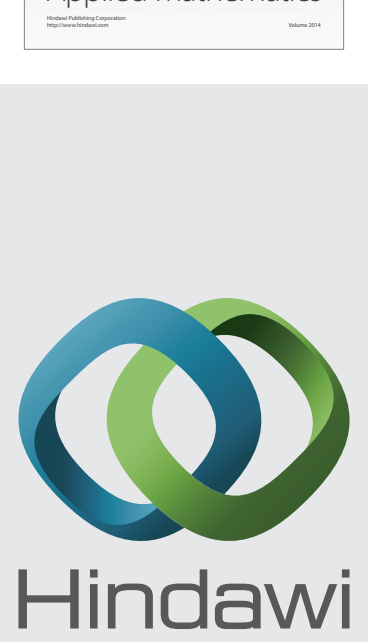

Submit your manuscripts at http://www.hindawi.com
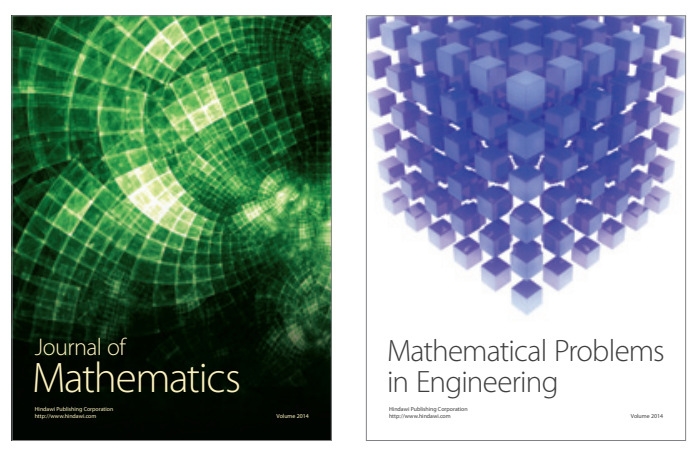

Mathematical Problems in Engineering
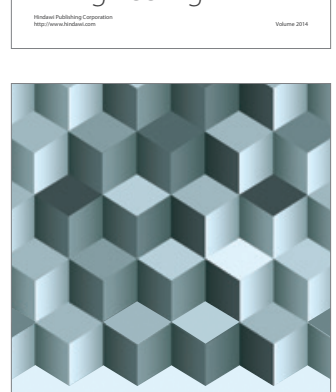

Journal of

Function Spaces
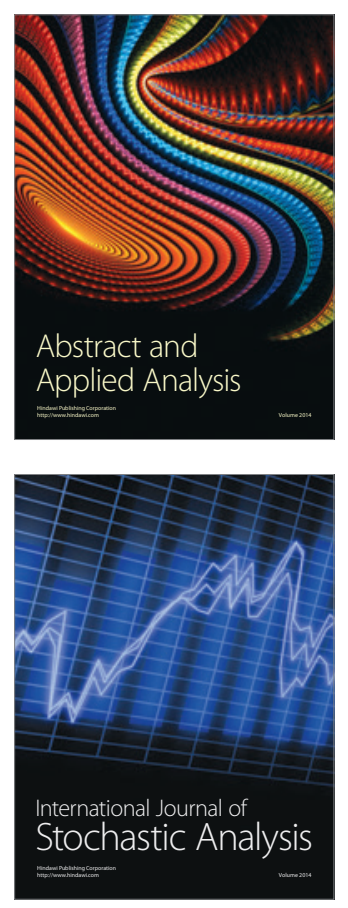

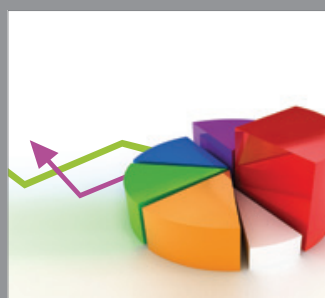

ournal of

Probability and Statistics

Promensencen
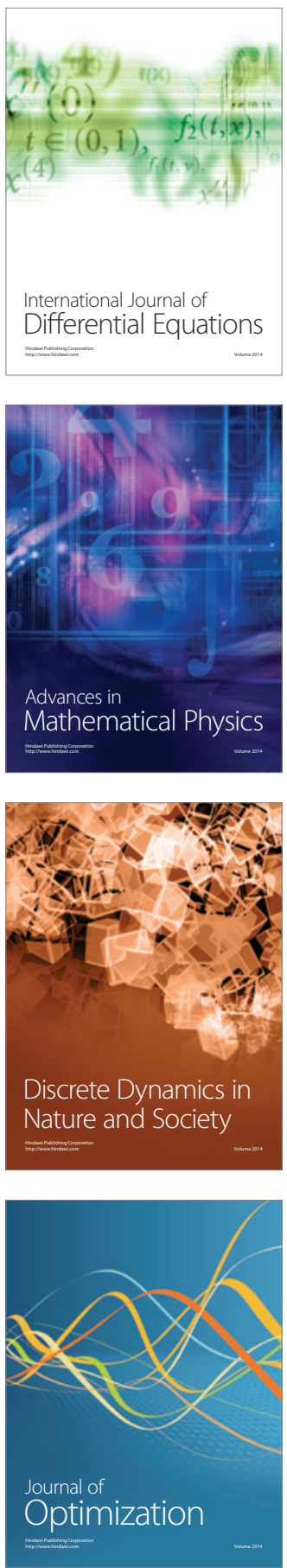